\title{
Pendulum experiments with three modern electronic devices and a modeling tool
}

\author{
Wing-Kwong Wong • Tsung-Kai Chao • \\ Pin-Ren Chen • Yunn-Wen Lien • Chao-Jung Wu
}

Received: 31 August 2014/Revised: 12 January 2015/Accepted: 16 January 2015/

Published online: 17 February 2015

(C) Beijing Normal University 2015

\begin{abstract}
Traditional physics labs in high school suffer from slow data acquisition so that some dynamic behavior of variables could be hidden from students. Modern electronic devices such as Lego Mindstorms NXT, smartphones and Arduino can acquire data at a fast rate and can be used to measure dynamic variables with reasonable precision in physics experiments. A case in point is the changing angle of a pendulum experiment. With a tool called InduLab, students in three groups using the mobile devices mentioned above in pendulum experiments collected data and built their models with the data. Experimental results showed that the Arduino group achieved the highest success rate of building correct models, followed by the smartphone group and then the NXT group. The results indicate that modern lowcost electronic devices can be used to improve physics labs in high school.
\end{abstract}

\footnotetext{
W.-K. Wong · P.-R. Chen ·

Department of Electronic Engineering, National Yunlin University of Science and Technology,

Douliu, Taiwan

e-mail:wongwk@yuntech.edu.tw

P.-R. Chen

e-mail: M10113212@yuntech.edu.tw

T.-K. Chao $(\bowtie)$

Graduate School of Engineering \& Technology, National Yunlin University of Science and Technology, Douliu, Taiwan

e-mail: g9810815@yuntech.edu.tw

Y.-W. Lien •

Department of Psychology, National Taiwan University, Taipei, Taiwan

e-mail: ywlien@ntu.edu.tw

C.-J. Wu .

Department of Educational Psychology \& Counseling, National Taiwan Normal University, Taipei, Taiwan

e-mail: cjwu@ntnu.edu.tw
} 
Keywords Physics experiments · Model-building tool $\cdot$ Mobile devices · Arduino $\cdot$ Smartphones $\cdot$ NXT

\section{Introduction}

In physics education, different teaching methods can be used depending on the topics. Laboratory work has been used to demonstrate various physical phenomena and remains the mostly used method (Kaya and Boyuk 2011). Physics theory can be abstract and difficult for students to comprehend. Fortunately, physics experiments can be performed individually or in groups to help students turn abstract theory into concrete data and data plots (Staeck 1995). Laboratory methods encourage students to apply their knowledge instead of just memorizing it. With laboratory sessions, students learn to improve their skills to comprehend the concepts deeper, apply them to daily life, and develop better attitudes toward physics lessons (Algan 1999).

However, in the high schools in Taiwan, physics laboratory classes are not compulsory because they are often sacrificed for the textbook classes, which are believed to lead to better student grades in public exams. Usually, teachers just do lab demonstrations instead of letting the students do the labs. Once a while, the students might be able to do a couple of labs in their three years of high school. Another problem in physics lab is outdated lab equipment. For example, in a pendulum experiment, students might measure the time for ten swings and are asked to get the average time for one swing by assuming all swings have equal periods. But this is a big assumption and students might not even realize this is an assumption. This problem can be solved with the introduction of microcomputerbased laboratory (MBL) (Yun-Ju Chiu 2008).

MBL is a well-known approach to learning sciences. It makes use of a computer or a microcontroller to do data logging. Then the students can plot the logged data on a monitor and fit the data with a curve specified as an equation (a mathematical model) with some plotting software. They can revise the model repeatedly until the fitted curve produces the least error.

Russell (2002) summarizes a number of advantages attributed to MBLs. First of all, the computer provides a number of advantages, including the data logging speed, the huge size of data storage, immediate display of processed data, data capture within a tiny fraction of a second or a number of days, data captured by multiple sensors at the same time, etc. These advantages also benefit the students. High-tech equipment motivates students to learn, the lab procedure is made easier for the less confident students, and waiting time is reduced in collecting and analyzing data. Finally, there are a number of pedagogical gains. For example, the computer provides multiple representations of data, such as the visual motion of an object and its displacement/velocity/acceleration graphs; students can investigate and repeat and control experiments individually and in group; lab activities train students on graphing skills and graph interpretation.

Compared to doing pure calculation with just physics theory, physics experiments can be a hands-on activity with more fun. Physics experiments and 
related tasks are practices of scientific inquiry, which is a process of investigation leading to some conclusion expressed mathematically. A common approach of scientific inquiry is microworld, e.g., with Papert's turtle graphics of Logo programming (Papert 1980), where students manipulate the values of some variables or do programming to explore and enhance their understanding of geometric concepts by observing the data produced by their manipulation. A pedagogy of scientific inquiry is also illustrated by Frideriksen and White (2005) with a computer-assisted learning method called ThinkerTools.

While microworld and ThinkerTools focus on the process of scientific inquiry, they differ from empirical physics experiments in that their inquiry results are based on computer simulation. Despite this difference, both simulated and empirical inquiries share the same scientific method with six steps:

1. Question: Construct a research question.

2. Hypothesize: Generate assumptions that might answer the question.

3. Investigate: Do experiments and collect the resulting data.

4. Analyze: Examine the resulting data to check against their hypotheses.

5. Model: Generate a mathematical model to explain the data.

6. Evaluate: Determine whether the model is correct and produce new hypotheses or new research questions.

In a traditional pendulum experiment in high school, students use a stop watch to time a number of swing periods and then compute the average period, assuming that every period consumes the same amount of time. Wong et al. (2011) reported a pendulum experiment using an Android smartphone as the mass of the pendulum. Wong et al. (2012) reported a similar pendulum experiment using an NXT instead of a smartphone. A pendulum was set up with a light, thin wooden rod, and a cell phone (or NXT) as the mass at the bottom of the rod. There was a hole at the top of the rod with a plastic axle through the hole and attached firmly to a wall so that the rod can pivot freely about the axle.

As the pendulum swung, a running application of the phone recorded the angular position and the corresponding time of the phone made with the vertical line through the point of attachment. The data plot showed a harmonic motion with decreasing amplitude. In this plot, the period of each wave can be measured and students can judge for themselves whether the swing periods stay the same within a certain time. After collecting sufficient data, students can then build a mathematical model relating the period $T$ of the pendulum and its length $L$. This is an important step where the predicted plot produced by the model should be drawn on top of the plot of the data collected from a real experiment. Then the error between the predicted plot and the data plot can be computed. Then the students can try to improve their model with better fitness and less error.

A common approach to math modeling is to use computational tools such as Excel (http://mmsphyschem.com/excelPhys1.htm) or Matlab (http://www. physics.purdue.edu/ hisao/book/www/Computational). However, the steep learning curve of these tools and the production of a model plot would be too timeconsuming for high school students to use such tools. In this study, the students were asked to use a model-building tool called InduLab, a previous version of which 
was presented in (http://140.125.32.150). All a student needs to do in testing a model is to enter a mathematical expression involving the dependent variable. Then the system will produce the predicted on a background of the data plot immediately. Then student can visually check how close or how far apart the two plots are from each other. Moreover, the error of fitness between the model and the data is computed and shown to the student immediately since the computation is straightforward and simple. Results from the empirical experiments in the current study could indicate how InduLab could help the model building process.

The above examples show that with high-speed data acquisition devices, students can examine physical phenomena in much greater details, like microbiologists studying bacteria with a microscope. This paper reports a study where high school students did pendulum experiments with three mobile devices of Arduino, smartphone, and NXT along with our model-building tool. The results would shed some light on how such devices and model-building tool can be used in similar physics experiments and how students feel about these inquiry tasks that are different from traditional labs.

This study attempts to answer three research questions. Can the students collect reasonable experimental data with the three devices? Can the students build reasonable models with InduLab with the data acquired by the three devices? How do the students react to this lab using the three devices and the modeling tool?

\section{Literature review}

\section{Science imagery}

The science imagery of a student will influence his attitude and motivation in learning science subjects (Benli et al. 2011; Kessels et al. 2006). Unfortunately, the science classes in school often lead to negative, unrealistic, and stereotypical images of science in students (Christidou 2011; Kessels et al. 2006; Osborne et al. 2003; Solbes and Traver 2003). Therefore, it is imperative to help students build positive science imagery. One resolution of this problem is inquiry-based learning for students in science classes, which is also a focus in the standard of national science education published by the National Research Council of the U.S. In scientific inquiry in school, students can improve their knowledge, learning skills, and strategies, and increase their interests and curiosities in learning about natural sciences (Wang et al. 2011).

\section{Mathematical modeling}

One objective of science is to build a model to describe the external world in order to explain the past and predict the future. According to Lesh and Doerr (2003), the perspective of mathematical modeling can promote the development of math instruction and learning. This perspective is a necessary strategy in cultivating the mathematical literacy in students in this new era of information technology. Sharing 
similar concern, Niss (2002) considers mathematical modeling as one of the eight most important math skills. This concern also attracts the attention of educators of mathematics (Houston et al. 1997; Blum 2002).

In the process of building a mathematical model, a student is asked to exercise his mathematical literacy in explaining and solving a problem that occurs in real life (Lesh and Doerr 2003; Lesh and Zawojewski 2007). In the end, the student must describe and solve the problem with mathematical methods and tools. In this process, when the student encounters difficulties, it is necessary for the instructor to provide strategic scaffolding to assist them (Yang and Lin 2006).

In this study, a model-building tool called InduLab is used by students in finding an equation whose curve fits the experimental data best. When a student enters a model to fit the experimental data, InduLab would immediately plot the model curve with the data and show the deviation of fitness. If a model produces less deviation, then it is a better model. This study will investigate how this numeric clue and the visual plot provide support for the student to revise and improve his model.

Pendulum experiment with Arduino at Queen's University

In a course Physics 350 at Queen's University, students use an Arduino to capture the angular position of a swinging physical pendulum equipped with a shaft encoder (http://www.physics.queensu.ca/ steve/phys350/Pendulum-quicksetupguide.pdf). The changing angular position of the pendulum is read by a shaft encoder (HEDS5500-A14, by Avago Technologies) and sent to an Arduino MEGA2560 which computes the data to produce an angular position. The Arduino periodically sends this position, along with a timestamp, to a computer through a USB connection (type B port on the Arduino, type A USB port on the computer).

\section{Pendulum sensor kit from IORodeo}

IORodeo, a commercial corporation providing lab technology, offers a pendulum sensor kit for classroom investigation of the physics pendulum (http://www.iorodeo. com/pendulum_lab). The kit has a pre-programmed Arduino and uses an LCD to display measurements such as the period of the pendulum in swing. The pendulum is mounted directly above a photogate sensor, and a pulley system allows simple adjustment of the length of the pendulum. The pendulum period is automatically computed by the provided computer program driving the Arduino. This automation is a disadvantage for our target high school students as we want them to learn how to measure the period of a wave and to examine whether the period stays the same as the pendulum swings. Too much automation that removes valuable learning experiences is one thing we should avoid in high school.

Period of a pendulum

In this experiment, an infrared barrier was used to detect the passage of the thread through a point (http://www.fisicayarduino.com.ar/period-of-a-pendulum/). 
Assembly is simple and consists of an infrared LED and an infrared phototransistor, with their respective strengths. It faces the LED and the transistor, so that the light emitted by the first delivered directly to the second. The collector of the transistor is connected to the junction 12 of Arduino, for use in digital form. When the light beam is interrupted, the phototransistor cuts off flow through the collector and Arduino interprets it as a pulse on LOW. If not, it is a high pulse.

The experimental data are based on locating the infrared barrier so that the wire crosses the beam twice during its journey. Each junction computes the time elapsed since the previous crossing. The oscillation period is the sum of two consecutive crossings time (trip time plus the back). This value is sent to the serial port for display on the console. Time is taken in microseconds, so the time must be divided by $10^{6}$ to convert to seconds, with a resolution of 6 decimals.

The experiment was completed by comparing the periods for various lengths of thread, and for various masses in the end of the thread, in this case checking that the period was unchanged. By increasing the mass, it was verified that the change did not affect significantly the length of the yarn.

\section{Pendulum pandemonium with LEGO Mindstorm NXT}

In this study, a pendulum experiment was taken by students at grade 11 (9-12 years old) (http://gk12.poly.edu/amps-cbri/pdf/pendulumRev6.pdf). Each group of students constructed a LEGO Mindstorm NXT setup that included a pendulum and a light sensor. The light sensor detected time instances when the pendulum's bob passed through a certain point as dips on a plot of measured light intensity. From these plots, students measured the period of the pendulum for different lengths of the pendulum rod. Next, they compared the experimentally determined values of the period to values calculated using a well-known formula. Finally, they changed the weight of the bob connected to the pendulum string and repeated the experiment to verify that the period of the pendulum is not affected by changes in the bob's weight. A discussion on the practical applications of pendulum technology and its history should be included as part of the post-activity assessment. The experimental procedure in this study included a construction phase, a programming phase, a prediction phase, a data collection phase, and experimental and theoretical comparison.

\section{Physics experiment}

Physics labs in high school are supposed to be fun activities compared to the studying of textbook theories. Compared with physics theories, labs provide opportunities for students to set up lab equipment and examine physical phenomena visually face to face. Theories are abstract and equation-driven, while labs are concrete and data-driven. Hopefully, fun labs help students understand the theories better and act like scientists with first-hand experiences. Unfortunately, outdated lab equipment with slow data acquisition rate might be an obstacle for students to 
examine closely the real physical phenomena. Therefore, this study proposes to use modern electronic devices that can measure and acquire physical data with a fast rate. An example lab in this study is the measurement of the changing angle of a swinging pendulum. The data acquisition devices this study tested included an Android smartphone, a Lego Mindstorms NXT, and an Arduino development board.

Following the six steps as in the Thinker Tools Inquiry Curriculum (White and Fereriksen 2000), students in this study can examine a target issue, make hypotheses, collect data from experiments, analyze the data, produce a model, validate the model, and can repeat the cycle until satisfactory results are obtained.

After students have collected experimental data, they can record the data, analyze them, and produce an equation to relate the measured variables with a software tool called InduLab. If a student finds that her equation does not fit the data well, she can modify her equation in order to improve the fitness.

A simple pendulum from any common physics textbook is shown in Fig. 1. It consists of a massless rod pivoted at its top end without any friction and a massive bob at the other end. The angle $\theta$ the rod makes with the vertical line through the pivot is the angle to be measured at a fast rate. The initial angle should be less than 10 degrees at the beginning of each experiment trial. In our pendulum lab, the rod used is a light, thin piece of wood, and the bob is a mobile device such as an Arduino board, a smartphone, or an NXT.

Different lengths were used for the pendulum and the corresponding periods were measured. After a set of data points of lengths and periods were obtained, students began to guess the length as a function of period. In textbook theory, the period $T$ is written as a function of the length $L$, since the length is an independent variable that can be manipulated and the period is a variable dependent on the length: $T=2 \pi \sqrt{\frac{L}{g}}$, where $g$ is the gravitational acceleration. This formula is not used directly in this study and instead the reverse of this relation is used, i.e., the length is a quadratic function of the period: $L=\frac{g}{4 \pi^{2}} T^{2}$. The reason is that our model-building tool restricts the functional form to be a regular polynomial that cannot involve the square root of a variable.

Fig. 1 A simple pendulum

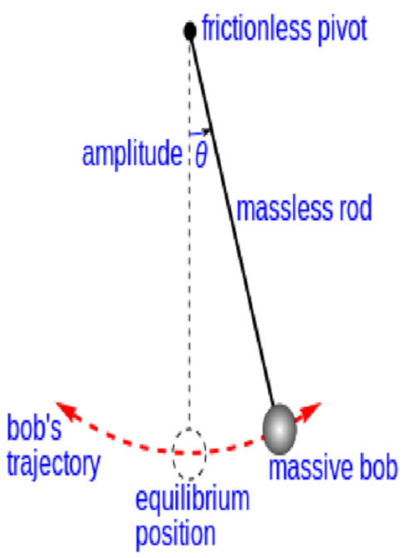




\section{Data acquisition devices and model-building tool}

Arduino board for data acquisition

A pendulum experiment can be set up using an Arduino development board and an accelerometer sensor. The particular board used in our study was Arduino ADK Rev3 Developer Edition 2, and the accelerometer was MX2125. A Bluetooth module was also used with Arduino to transmit to a PC the angle of the swinging pendulum and the time stamp when the angle was measured. The Arduino hardware was mounted with Lego bricks on a light, thin and flat wooden rod, whose top is freely pivoted on a hook attached to a wall. Besides serving as the data acquisition device, the Arduino hardware also served as the massive bob of the pendulum. The Arduino pendulum is shown in Fig. 2. The background of a paper protractor can show the initial angle of the pendulum. The pendulum with a smartphone and that with an NXT are shown in Figs. 3 and 4 respectively.

InduLab as a model-building tool

Once the file of experimental data in csv format is produced on a PC, a student will open the file with Microsoft Excel and obtain a plot of harmonic motion of angle versus time with decreasing amplitude (Fig. 5).

For each pendulum length, a student can measure the periods of ten waves, each of which can be the time between two neighboring maxima in the plot. The student then fills in the ten periods (in min) and the pendulum length (in mm). Now the student can tell from the data that the periods are more or less the same. Then the system will compute the average of the ten periods. Note that different lengths should be used for a total of six trials of the experiment. Finally, there will be six data points of periods and lengths. Figure 6 shows three pendulum lengths and their

Fig. 2 Arduino

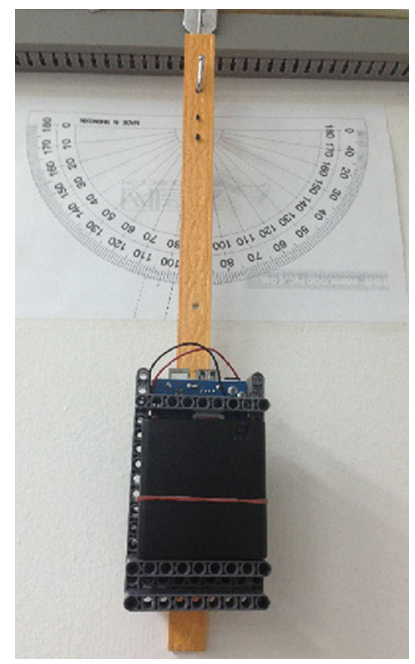


Fig. 3 Smartphone

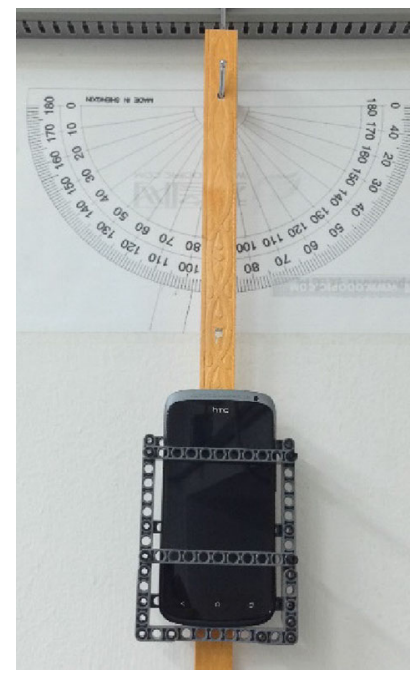

Fig. 4 NXT

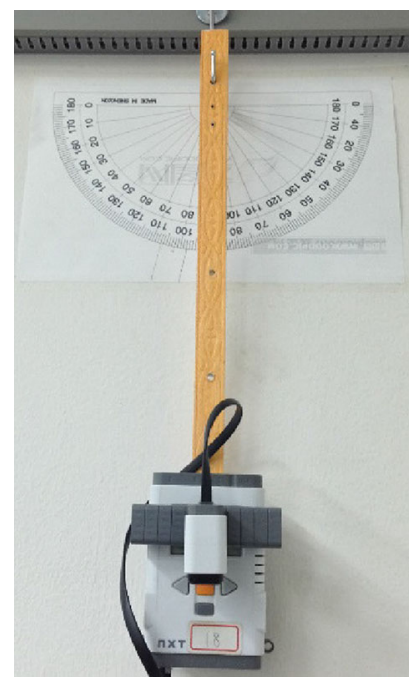

corresponding ten periods. The average of each set of ten periods is displayed at the bottom of the ten periods.

After six lengths and their corresponding averaged periods are obtained, each student can start to build their model that can predict the experimental data with minimum deviation. The student enters length $L$ as a function of period $T$. The mathematical expression is restricted to a regular polynomial with real coefficients. Each time he enters a function, the system will show a plot of experimental $T$ versus $L$ (diamond points) and also theoretical $T$ versus $L$ (circle points) predicted by the model. Also, a measure of deviation between the prediction and the actual data can show whether the model has less error than previous models. Figure 7 shows the last 


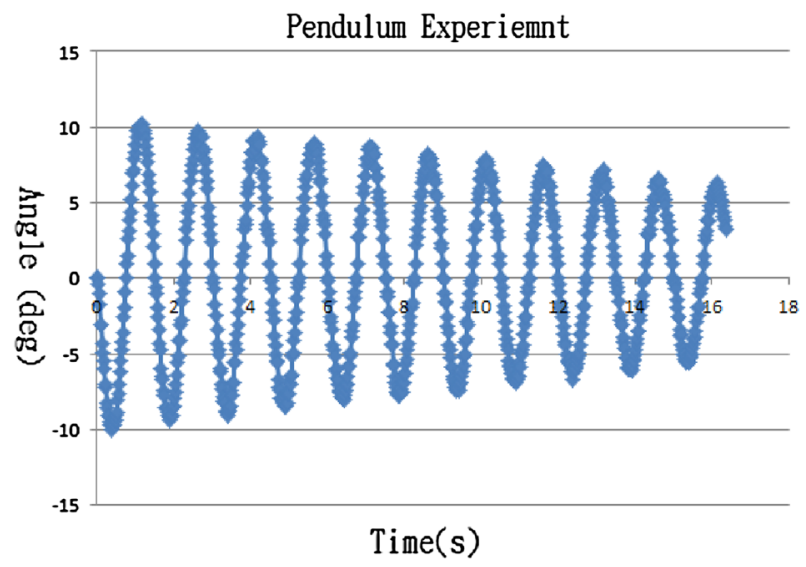

Fig. 5 Plot of the angle of a moving pendulum versus time

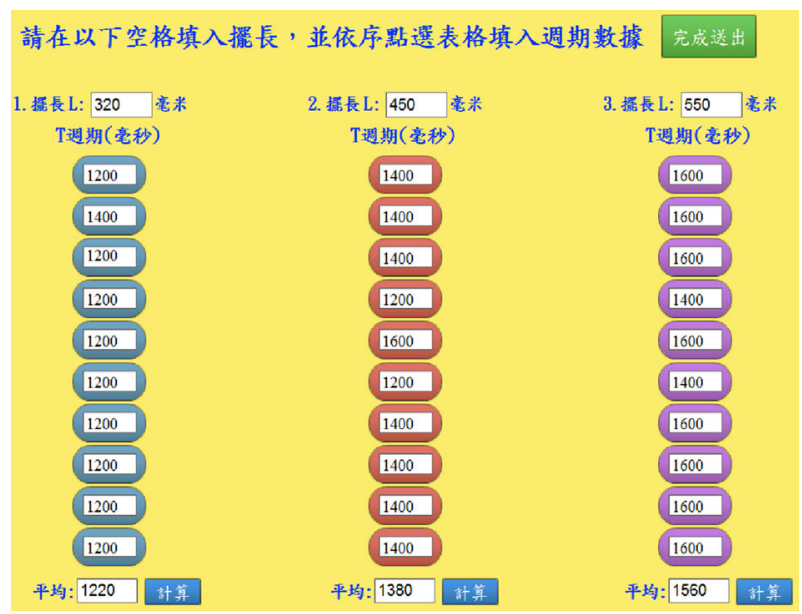

Fig. 6 Three pendulum lengths and ten of their corresponding periods

two models entered by a subject. The deviation was reduced from 35.6 to 22.7. The quadratic function with minimum deviation was chosen as a final model.

\section{Pendulum experiments and results}

The pendulum lab was done by 30 students in a high school in Yunlin County in four weeks. The students were divided into six groups, each of which had five members. Two groups used Arduino, two used smartphone, two used NXT. In the first week, the instructor introduced the pendulum experiment and its setup with the three mobile devices. In the second week, each group did six trials with different 


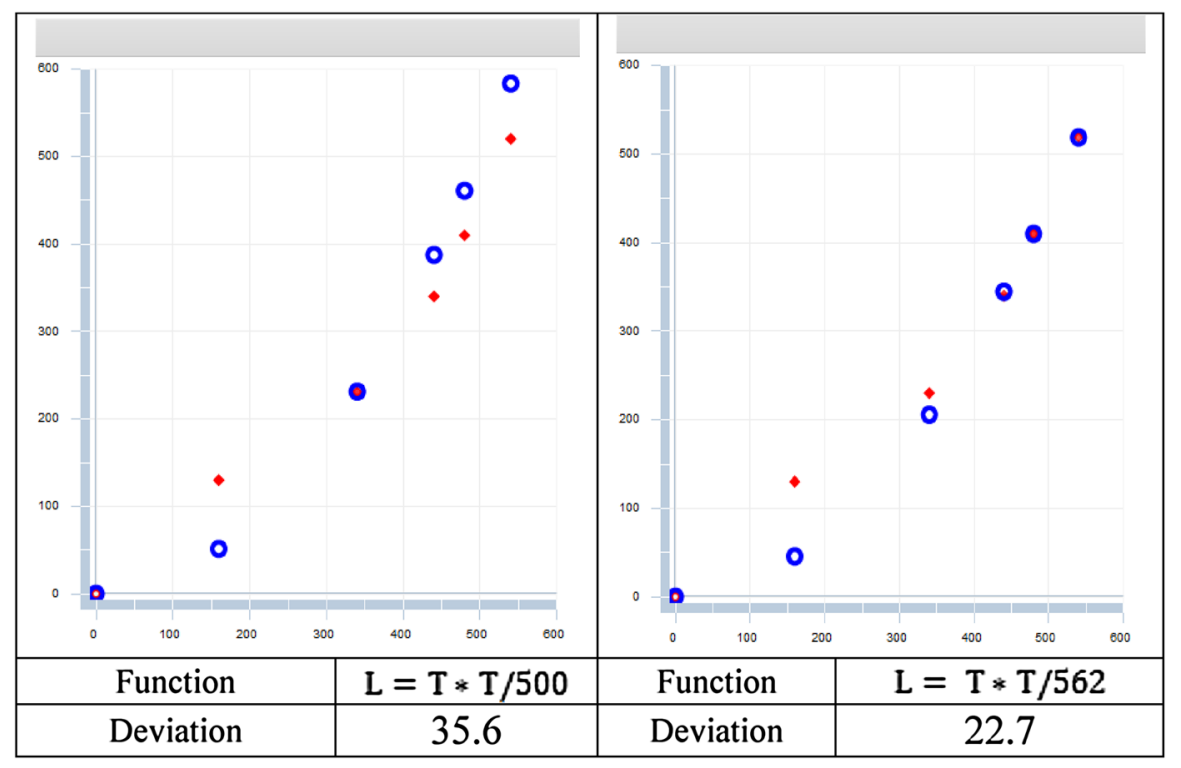

Fig. 7 The last two models entered by a student showed a reduction of deviation

pendulum lengths and collected the data. In the third week, each student worked on her own in InduLab to build a model with her group's data. In the last week, the instructor talked about the pendulum theory and suggested possible reasons to explain the differences among the models obtained by the students.

Textbook theory says that target formula of a simple pendulum is $L=\left(\frac{g}{4 \pi^{2}}\right) \times T^{2}$. Therefore, students whose final model was $L$ as a quadratic equation with a small error of fitness were considered successes.

The success rate for each device is shown in Table 1 . The success rates for the three groups were very different. The Arduino group succeeded with $80 \%$, the smartphone group $40 \%$, and the NXT group $10 \%$. The data acquisition rates for the three corresponding devices were 50, 50, and 5 points per second. The NXT group suffered a low success rate might very well be due to its relatively low acquisition rate. But the acquisition rate did not explain the different success rates between the Arduino group and the smartphone group. Table 2 shows the range of the g value of successful models using the three devices by using the theoretical model (with

Table 1 Success rates for three groups using different devices

\begin{tabular}{llllll}
\hline Device & Data acquisition rates & \#Successes & \multicolumn{2}{l}{ \#Subjects } & \multicolumn{2}{c}{ Success rate (\%) } \\
\cline { 3 - 5 } & & & Female & Male & \\
\hline Arduino & 50 & 8 & 5 & 5 & 80 \\
Smartphone & 50 & 4 & 5 & 5 & 40 \\
NXT & 5 & 1 & 2 & 8 & 10 \\
\hline
\end{tabular}


Table 2 Deviation of gravity ( $g$ ) based on each student's final model

\begin{tabular}{llll}
\hline Device & $g$ & & \\
\cline { 2 - 4 } & $9.7 \leq g<10$ & $11.5 \leq g<12.5$ & $15.5 \leq g$ \\
\hline Arduino (10 students) & 6 & 2 & 0 \\
Smartphone (10 students) & 2 & 0 & 2 \\
NXT (10 students) & 1 & 0 & 0 \\
\hline
\end{tabular}

Table 3 Specification of the sensors of the three devices

\begin{tabular}{lll}
\hline Device & Sensor & Specification \\
\hline Arduino & Memsic 2125 & Measures \pm 3 g on each axis \\
Smartphone & HTC one S gyroscope & Not provided by the vendor \\
NXT & NXT gyro sensor (NGY1044) & Measure up to $\pm 360^{\circ}$ per second of rotation \\
\hline
\end{tabular}

$g=9.8 \mathrm{~m} / \mathrm{s}^{2}$ ) of a simple pendulum as a reference. The results showed that the Arduino group produced $g$ values closer to the theoretical target of 9.8, trailing by the Smartphone group and then the NXT group. The hardware specification for each device's sensor is shown in Table 3.

Some of the models produced sequentially by a student $\mathrm{S}$ using the Arduino setup are shown in Table 4.

Consider the student $\mathrm{S}$ in the above table. In the beginning, $\mathrm{S}$ tested three linear models to fit the data, and the deviation was reduced to 269.37. After trying out a quadratic model temporarily, $\mathrm{S}$ returned to testing linear models with different values of coefficients. After some trials, $\mathrm{S}$ switched to cubic models. However, the minimum deviation obtained was 438.333. Later, S switched to quadratic models, starting at $\frac{T \cdot T}{500}$. After trying out different coefficients in many steps, S progressively reduced the deviation down to 30.676 with the model $L=\frac{T^{2}}{3950}-26$.

High school students are seldom trained in using Excel to build mathematical models. With Excel, it takes much more time for a student to generate and plot the data predicted by the student's model. In InduLab, the student just enters a polynomial expression involving the dependent variable. Then the system will compute all predicted data, plot them against the experimental data, and obtain the deviation of fitness. Because model checking was so easy to do that the student could quickly check the fitness of the model. This explained why the student in Table 4 tried so many models. This indicated that the tool was easy to use and did provide useful information for the model revision process.

The model can be compared to the theoretical model $L=\left(\frac{g}{4 \pi^{2}}\right) \times T^{2}$. After some algebraic simplification and adjustment of unit, $g=9.98 \mathrm{~m} / \mathrm{s}^{2}$, which was close to the theoretical $g$ value of $9.8 \mathrm{~m} / \mathrm{s}^{2}$. This indicated the student's final model was quite close to the theoretical model. 


\section{Conclusion}

Traditional Physics experiments can be improved with two great tools: low-cost modern electronic devices and a computer-assisted model-building environment. This study shows how to do the pendulum experiment with three devices, including Arduino, smartphone, and Lego Mindstorms NXT. Smartphones are usually treated as toys by students in school, and NXTs are usually treated as robotic toys and they are seldom thought as learning tools in physics experiments. Arduino platforms are more widely accepted as a learning tool at college level and mostly by electronics hobbyists. Its potential as a data acquisition device in physics experiments is proposed by this and other studies as cited above. The results of this study presented above indicated that students were able to collect data with all three devices, and the data plots showed simple harmonic motion with decreasing amplitude. The data collected with Arduino devices and smartphones were better for the following modeling task since their sampling rates were faster than that of NXT devices.

After the data were collected in experiments and stored in a file on a PC, students used the data to build a mathematical model of the physical phenomenon of the experiment. A tool called InduLab was used for this purpose. In a pendulum experiment, students simply expressed the length of the pendulum as a function of its period. The predicted values were plotted on the same graph as the experimental data and their deviation were computed. Both the visual plot and the automatically computed deviation helped the students to bring the prediction closer to the experimental data. Empirical results presented above indicated that students using InduLab were able to refine their models to fit the data better with decreasing deviation. However, their final models might not fit the data well if they used linear models instead of quadratic models. In other words, the modeling tool does not promise to produce successful models. That remains to be the responsibility of the student.

For the pendulum experiment, the Arduino group achieved the highest rate of building successful models judged by the theory in textbook. The smartphone group came in second followed by the NXT group. Both Arduino and the smartphone had a high data acquisition rate of 50 points per second, while NXT had a relatively low rate of five points per second. This might explain the low success rate of the NXT group. But it was intriguing why the Arduino group was doing better than the smartphone group. A simple explanation was that the better result was due to better performance efforts of the Arduino group. From the current preliminary results, it should be fair to conclude that the knowledge and skills of a student, the measurement accuracy of the hardware, and the modeling tool all contribute to the success of a model. A reasonable measurement accuracy of the data logging device is necessary to collect experimental data. With poor data, a successful model would be impossible to obtain. Students must also possess sufficient mathematical maturity in order to produce a successful model from good data. Further empirical experiments with more subjects can shed more light on the issues raised by this study.

Most students showed their enthusiasm in almost all lab tasks and improved their task skills as time passed. They were especially excited about the use of 
Table 4 The models produced by a student

\begin{tabular}{|c|c|c|c|}
\hline Step & Deviation & Model & Leading term \\
\hline 1 & 2311 & $\mathrm{~T} * 2$ & $\mathrm{~T}$ \\
\hline 2 & 285.593 & $\mathrm{~T}^{*} 1 / 9$ & \\
\hline 3 & 269.37 & $\mathrm{~T} * 5 / 9-56$ & \\
\hline 4 & 237821.333 & $\mathrm{~T} * \mathrm{~T}-1906940$ & $\mathrm{~T}^{2}$ \\
\hline 5 & 877.333 & $\mathrm{~T}-59$ & $\mathrm{~T}$ \\
\hline \multirow[t]{2}{*}{6} & 836.333 & $\mathrm{~T}-100$ & \\
\hline & $\ldots$ & $\ldots \ldots$ & \\
\hline 42 & 394902.9 & $\mathrm{~T} / 8 * 2-1 / 9 * 10-10 / 555 * 75+100 * 3950$ & \\
\hline \multirow[t]{2}{*}{43} & 137369.5 & $\mathrm{~T} / 8 * 2-1 / 9 * 10-10 / 555 * 75+100 * \mathrm{~T}$ & \\
\hline & $\ldots$ & $\ldots$ & \\
\hline 50 & $2.67 \mathrm{E}+09$ & $\mathrm{~T}^{*} \mathrm{~T} * \mathrm{~T}$ & $\mathrm{~T}^{3}$ \\
\hline 51 & 17366.6 & $\mathrm{~T}^{*} \mathrm{~T} * \mathrm{~T} / 150000$ & \\
\hline \multirow[t]{2}{*}{52} & 438.333 & $\mathrm{~T} * \mathrm{~T} * \mathrm{~T} / 15000000000000000$ & \\
\hline & $\ldots$ & $\ldots$ & \\
\hline \multirow[t]{2}{*}{99} & 13770.85 & $\mathrm{~T} * \mathrm{~T} * \mathrm{~T} / 5000000-120-\mathrm{T} * 10$ & \\
\hline & $\ldots$ & $\ldots$ & \\
\hline 102 & 3376.423 & $\mathrm{~T} * \mathrm{~T} / 500$ & $\mathrm{~T}^{2}$ \\
\hline 103 & 37709.23 & $\mathrm{~T} * \mathrm{~T} / 50$ & \\
\hline 104 & 381037.3 & $\mathrm{~T} * \mathrm{~T} / 5$ & \\
\hline \multirow[t]{2}{*}{105} & 1906940 & $\mathrm{~T} * \mathrm{~T}$ & \\
\hline & $\ldots$ & $\ldots$ & \\
\hline 125 & 33.744 & $\mathrm{~T} * \mathrm{~T} / 3900-26$ & \\
\hline 126 & 31.397 & $\mathrm{~T} * \mathrm{~T} / 4000-26$ & \\
\hline 127 & 34.305 & $\mathrm{~T} * \mathrm{~T} / 4100-26$ & \\
\hline 128 & 30.676 & $\mathrm{~T} * \mathrm{~T} / 3950-26$ & \\
\hline
\end{tabular}

smartphones as data logging and learning devices because most of them took the smartphones for granted as only toys to play with. They also indicated that the model-building tool was simple to use. The supervising physics teacher also expressed keen interests in these data logging devices and the model-building tool. In short, this study indicated that this MBL approach with modern electronic devices was well accepted by both students and teacher.

Besides doing the pendulum experiment, the students also used the same electronic devices with an ultrasonic sensor in other experiments, including free-fall projectile, vertical free-fall projectile, slope motion, the second Newton's law. This shows that the devices are really general-purpose and can work in various labs.

High school physics teachers are already very busy in preparing students for national aptitude tests and might not be aware of the capability of low-cost modern electronic devices. So one contribution of this study is that we have shown how high school students can use modern electronic devices in physics experiments, instead 
of using the traditional equipment with low rate of data acquisition. The second contribution is that InduLab serves as an innovative tool of model construction that fits seamlessly in physics labs and might help to bring back the play-scientist fun of rediscovery that is somehow lost in physics education for many years in high school.

Acknowledgments This study is supported by the Department of International Cooperation and Science Education, Ministry of Science and Technology, Taiwan under contract 101-2511-S-224-001- and 1022511-S-224-002-MY2.

\section{References}

A pendulum sensor kit from a commercial company IORodeo Smart Lab Technology. http: //www.iorodeo.com/pendulum_lab.

Algan, Ş. (1999). The Effect of physics lessons supported by lab experiments to student's success and modern mathematics and science programmes conducted in Turkey between 1962-1985. Unpublished master's thesis, Gazi University Institute of Science, Ankara.

Benli, E., Dökme, İ., \& Sarikaya, M. (2011). The effects of technology teaching materials on students' image of scientists. Procedia Social and Behavioral Sciences, 15, 2371-2376.

Blum, W. (2002). ICMI study 14: Applications and modeling in mathematics education-Discussion document. Educational Studies in Mathematics, 51(1), 149-171.

Christidou, V. (2011). Interest, attitudes and images related to science: Combining students' voices with the voices of school science, teachers, and popular science. International Journal of Environmental and Science Education, 6(2), 141-159.

Computational Physics using MATLAB. http://www.physics.purdue.edu/ hisao/book/www/ Computational Physics using MATLAB.pdf.

Course phys350 of Queen's University experiment document. http://www.physics.queensu.ca/ steve/ phys350/Pendulum-quicksetupguide.pdf.

Houston, S. K., Blum, W., Huntley, I., \& Neill, N. T. (Eds.). (1997). Teaching and learning mathematical modelling. Chichester: Albion Pub.

InduLab: http://140.125.32.150.

Kaya, H., \& Boyuk, U. (2011). Attitudes towards physics lessons and physical experiments of the high school students. European Journal of Physics Education, 2(1), 38-49.

Kessels, U., Rau, M., \& Hannover, B. (2006). What goes well with physics? Measuring and altering the image of science. British Journal of Educational Psychology, 74(4), 761-780.

Lesh, R., \& Doerr, H. (2003). Foundations of a models and modelling perspective on mathematics teaching, learning, and problem solving. In R. Lesh \& H. M. Doerr (Eds.), Beyond constructivism: Models and modeling perspectives on mathematics problem solving, learning, and teaching (pp. 334). Mahwah, NJ: Lawrence Erlbaum Associates Inc.

Lesh, R., \& Zawojewski, J. S. (2007). Problem solving and modeling. In F. Lester (Ed.), The second handbook of research on mathematics teaching and learning (pp. 763-804). Charlotte, NC: Information Age Publishing.

Niss, M. (2002). Mathematical competencies and the learning of mathematics: The Danish KOM project. Retrieved October 23, 2014, from http://www7.nationalacademies.org/mseb/mathematical_ competencies_and_the_learning_of_mathematics.pdf.

Osborne, J., Simon, S., \& Collins, S. (2003). Attitude towards science: A review of the literature and its implications. International Journal of Science Education, 25(9), 1049-1079.

Papert, S. (1980). Computer-based microworlds as incubators for powerful ideas. In R. Taylor (Ed.), The computer in the school: Tutor, tool, tutee (pp. 203-210). New York: Teacher's College Press.

Pendulum Pandemonium with LEGO Mindstorm NXT. http://gk12.poly.edu/amps-cbri/pdf/ pendulumRev6.pdf.

Period of a pendulum. http://www.fisicayarduino.com.ar/period-of-a-pendulum/.

Physics I \& Excel. http://mmsphyschem.com/excelPhys1.htm.

Russell, D. (2002). Computers in physics instruction: Students' interactions in a constructivist microcomputer-based laboratory. Doctoral thesis, Queensland University of Technology, Australia. 
Solbes, J., \& Traver, M. (2003). Against a negative image of science: History of science and the teaching of physics and chemistry. Science and Education, 12(7), 703-717.

Staeck, L. (1995). Perspectives for biological education-challenge for biology instruction at the end of the 20th century. Hacettepe University Journal of Education, 11, 29-35.

Wang, J. R., Tsay, R. F., Lee, K. P., Lin, S. W., Kao, H. L., \& Huang, B. Y. (2011). A meta-analysis of inquiry-based instruction on student learning outcomes in Taiwan. The Asia-Pacific Education Researcher, 20(3), 534-542.

White, B. Y., \& Fereriksen, J. R. (2000). Technological tools and instructional approaches for making scientific inquiry accessible to all. In M. J. Jacobson \& R. B. Kozma (Eds.), Innovations in science and mathematics education: Advanced designs for technologies of learning (pp. 321-359). Mahwah, NJ: Lawrence Erlbaum Associates.

White, B., \& Frederiksen, J. A. (2005). A theoretical framework and approach for fostering metacognitive development. Educational Psychologist, 40(4), 211-223.

Wong, W. K., Chao, T. K., Lien, Y. W., \&Wu, C. J. (2012). Rediscovering scientific laws in high school physics labs with mobile devices, Proceedings of International Conference on Computers in Education, Asia-Pacific Society for Computers in Education, Singapore.

Wong, W. K., Xu, J. M., Chao, T. K. (2011). Using Android mobile device for physics experiments and inquiry, International Conference on Computers in Education, 2011/11/28-2011/12/02, APSCE, Chiangmai.

Yang, K. L., \& Lin, F. L. (2006). Exploring the scaffolding strategies of inserting mathematical modeling into teaching of secondary mathematics and the latent mechanism of promoting teachers' reflection. Chinese Journal of Science Education, 14(5), 517-543.

Yun-Ju Chiu (2008f). Learning by doing: Implications for Instruction in Simple Hands-On Experiments, 發表於2008年海峽兩岸物理基礎課程教學研討會, 華東理工 大學主辦, 上海 $(2008 / 5 / 23 \sim 25)$. 論 文刊登於《華東理工大學學報》, 第34卷增刊, 152 157 頁, 2008年6月.

Wing-Kwong Wong is an Associate Professor in the Department of Electronic Engineering, National Yunlin University of Science \& Technology, Taiwan. His research interests include E-learning, robotics, embedded systems, and computational design.

Tsung-Kai Chao is a PhD student in the Graduate School of Engineering Science and Technology, National Yunlin University of Science \& Technology, Taiwan. His research interests include science inquiry with technology products, E-learning, and mobile game design.

Pin-Ren Chen was a graduate student in the Department of Electronic Engineering, National Yunlin University of Science \& Technology, Taiwan. Now he works as an application engineer in Taiwan.

Yunn-Wen Lien is an associate professor in the Department of Psychology, National Taiwan University, Taiwan. Her research interests include rule discovery, creativity, causal reasoning and mindfulness.

Chao-Jung Wu is a professor in the Department of Educational Psychology and Counseling, National Taiwan Normal University, Taiwan. Her Research interests include mathematical reasoning, and eye movements in reading illustrated text. 